\title{
FINITE QUASI-INJECTIVE GROUPS
}

\author{
by DENNIS BERTHOLF and GARY WALLS
}

(Received 6 July, 1977; revised 8 October, 1977)

It is well known that the category of finite groups has no non-trivial injective objects. In general, a group is said to be quasi-injective if for every subgroup $H$ of $G$ and homomorphism $f: H \rightarrow G$ there exists an endomorphism $F: G \rightarrow G$ such that $\left.F\right|_{H}=G$. In other words, a group is quasi-injective whenever each homomorphism from a subgroup into the group can be extended to the whole group.

In this paper we completely characterize the class of finite, quasi-injective groups. In Fuchs [2] all quasi-injective, abelian groups are determined. It follows from this result that the finite, abelian, quasi-injective groups are those abelian groups in which each Sylow subgroup is a direct product of isomorphic cyclic groups. We call such groups, homocyclic groups. The notation will be standard and can be found in [3].

We first note the following lemmas.

LемMA 1. (i) A direct factor of a quasi-injective group is quasi-injective.

(ii) A fully-invariant subgroup of a quasi-injective group is quasi-injective.

(iii) If a fully-invariant subgroup of a quasi-injective group has one element of order $n$, it contains all the elements of order $n$.

Proof. (i) Let $H$ be a factor of the quasi-injective group $G$. Let $i: H \rightarrow G$ be the inclusion map and suppose $p: G \rightarrow H$ is such that $p i=1_{H}$. Let $L \subseteq H$ and suppose $f: L \rightarrow H$ is a homomorphism. We must show how to extend $f$ to all of $H$. Since $G$ is quasi-injective we can find $F_{1}: G \rightarrow G$ such that $\left.F_{1}\right|_{L}=i f$. We define $F: H \rightarrow H$ by letting $F=\left.p F_{1}\right|_{H}$. Then, if $h \in L, F(h)=p F_{1}(h)=p(i f(h))=f(h)$. Hence $\left.F\right|_{L}=f$, as required.

(ii) This is clear.

(iii) Let $H$ be a fully-invariant subgroup of the quasi-injective group $G$. Let $x \in H$ have order $n$. By the quasi-injectivity of $G$, there exists an endomorphism $F: G \rightarrow G$ such that $F(x)=y$, where $y$ is any element of order $n$. But then $y=F(x) \in H$ as $H$ is fully-invariant. This completes the proof.

Lemma 2. Suppose $G=H \times K$, where $(|H|,|K|)=1$. Then $G$ is quasi-injective if and only if both $H$ and $K$ are.

Proof. $(\Rightarrow)$ This follows from Lemma 1(i).

$(\Leftarrow)$ Suppose that $H$ and $K$ are quasi-injective groups. Suppose, also, that $L$ is a subgroup of $G$ and that $f: L \rightarrow G$ is a homomorphism. Since $(|H|,|K|)=1$, we have $L=(L \cap H) \times(L \cap K)$. Since $H, K$ are quasi-injective, there exist endomorphisms $F_{1}: H \rightarrow H$ and $F_{2}: K \rightarrow K$ such that $\left.F_{1}\right|_{L \cap H}=\left.f\right|_{L \cap H}$ and $\left.F_{2}\right|_{L \cap K}=\left.f\right|_{L \cap K}$.

It is then clear that the map $F: G \rightarrow G$ defined by $F(h k)=F_{1}(h) F_{2}(k), h \in H, h \in K$, is an endomorphism of $G$ such that $\left.F\right|_{L}=f$, as required.

Glasgow Math. J. 20 (1979) 29-33. 
REMARK. It is clear that $Q$, the quaternion group of order 8 , is quasi-injective. The fact that $Q \times Q$ is not quasi-injective shows that the above result is false without the assumption about the orders of $H$ and $K$.

THEorem 3. A finite, quasi-injective group is supersolvable.

Proof. Suppose the theorem is not true for the group $G$; then we can choose $H \triangleleft G$ minimal with respect to $G$ having a noncyclic chief factor $H / K$. Then $p^{2}|| H / K \mid$ for some prime $p$. Let $H_{p} / K \in \operatorname{Syl}_{p}(H / K)$; then there is an $f: H_{p} \rightarrow G$ with kernel $K_{p} \supset K$ and $\left|H_{\mathrm{p}} / K_{\mathrm{p}}\right|=p$. Since $f$ extends to $G$, there is an $M \triangleleft G$ such that $M \cap H_{\mathrm{p}}=K_{\mathrm{p}}$ and hence $H \supset M \cap H \supset K$, contrary to $H / K$ being a chief factor.

The next theorem is very useful in determining the structure of quasi-injective groups.

THEOREM 4. Let $G$ be a finite quasi-injective group. Then if $H$ is a subnormal subgroup of $K, K \subseteq G$, we have $H \triangleleft K$.

Proof. Let $H \triangleleft H_{1} \triangleleft \ldots \triangleleft H_{n-1}=L \triangleleft K$. Proceeding by induction on $n$, we suppose that $H \triangleleft L$. If $H \triangleleft K$ then there is a $k \in K$ such that $H^{k} \neq H$. Let $U=H H^{k} \subseteq L$. $U / H$ has a quotient of order $p$ and so there is an $M \triangleleft G$ such that $U \supset U \cap M \supseteq H$. But then $M \supseteq H^{x}$ and so $M \supseteq H H^{x}=U$. This is a contradiction.

This theorem has the following immediate corollary.

COROLLARY. Every nilpotent subgroup of a finite, quasi-injective group is a Dedekind group.

Lemma 5. Suppose that $G$ is a quasi-injective group and $S$ is a Sylow p-subgroup of $G$ for some prime $p$. Then all the elements of order $p$ in $S$ have the same height in $S$ (i.e. they are contained in isomorphic maximal cyclic subgroups of $S$ ).

Proof. Suppose $|x|=p$ and $x \in S$. From the definition of quasi-injective, it is easy to see that all the elements of order $p$ have the same $p$-height in $G$. Hence suppose $y \in G$ is a $p$-element such that $x \in\langle y\rangle$ and $\langle y\rangle \subseteq Q$, where $Q$ is a Sylow $p$-subgroup of $G$. Thus there exists $z \in G$ such that $x^{z} \in\langle y\rangle^{z}=\left\langle y^{z}\right\rangle \subseteq Q^{z}=S$. If $\left\langle x^{z}\right\rangle \neq\langle x\rangle$ we get a contradiction as in Theorem 4. (Note $\langle x\rangle\left\langle x^{z}\right\rangle$ exists as $S$ is a Dedekind group.) Hence $\langle x\rangle=\left\langle x^{z}\right\rangle$. Thus $x \in\left\langle x^{z}\right\rangle \subseteq\left\langle y^{2}\right\rangle \subseteq S$. Hence $x$ has the same $p$-height in $S$ that it had in $G$. This completes the proof.

COROLlary. A Sylow p-subgroup of a quasi-injective group is either a homocyclic p-group or a quaternion group of order 8.

Proof. Let $S$ be a Sylow p-subgroup of $G$. Since $S$ is a Dedekind group, $S$ is abelian or the direct product of a quaternion group of order 8 with an elementary abelian 2-group (cf. [5, Theorem 9.7.4]). By the above lemma, $S$ has the desired form.

The above result implies that if a Sylow $p$-subgroup $S$ of a quasi-injective group is non-abelian then $p=2$ and $S$ is a quaternion group of order 8 . The next theorem shows that in this case $S$ must be a direct factor of $G$. 
THEOREM 6. If the Sylow 2-subgroup of the quasi-injective group $G$ is $Q$, the quaternion group of order 8 , then $G=Q \times H$ where $H$ is a quasi-injective group of odd order.

Proof. By the Frattini argument, $G=G^{\prime} N_{G}(Q) .\left(Q \in \operatorname{Syl}_{2}\left(Q G^{\prime}\right), Q G^{\prime} \triangleleft G\right.$.) Therefore, if $Q \triangleleft G$, there is a $p$-element $x \in G^{\prime}$ such that $x \notin N_{G}(Q)$. Since $G$ is supersolvable, $G^{\prime}$ is nilpotent. Hence, by Theorem $4,\langle x\rangle \triangleleft G$.

The group of automorphisms induced in $\langle x\rangle$ by $Q$ is cyclic and hence of order 2 . Thus $C_{Q}(x)$ is cyclic of order 4 . This being the case for each Sylow 2-subgroup of $G$ we have that each element of order 2 centralizes $x$. The natural map $f:\langle x\rangle C_{Q}(x) \rightarrow\langle x\rangle$ can be extended to a homomorphism $F: G \rightarrow G$. If $M=\operatorname{ker} F$ then $M \cap\langle x\rangle C_{Q}(x)=C_{Q}(x)$.

Now $M \cap\langle x\rangle Q \triangleleft\langle x\rangle Q$ and contains $C_{Q}(x)$. As $M \cap\langle x\rangle=1, M \cap\langle x\rangle Q \subseteq C_{Q}(x)$. Thus $M \cap\langle x\rangle Q=C_{Q}(x)$. Therefore im $F$ contains the subgroup $(\langle x\rangle Q) M / M$ which is isomorphic to $\langle x\rangle Q / C_{Q}(x)$. But this contains a non-central element of order 2 . This contradiction implies that $Q \triangleleft G$. The Sylow tower property of $G$, and Lemma 2 then give the result.

The above result and Lemma 2 have thus reduced the discussion to groups all of whose Sylow subgroups are abelian. The following sequence of results leads to a characterization of the finite quasi-injective groups all of whose Sylow subgroups are abelian. Thus in Lemmas 7, 8, 9 we will be assuming that $G$ is a quasi-injective group all of whose Sylow subgroups are abelian.

Lemma 7. Let $G$ be a finite quasi-injective group. Then $G^{\prime}$ is a Hall $\pi$-subgroup of $G$ and there exists $H \subseteq G$ such that $G=G^{\prime} H$ with $G^{\prime} \cap H=1$. Furthermore, $G^{\prime}$ and $H$ are homocyclic abelian groups and $G^{\prime} \cap Z(G)=1$.

Proof. From Theorem 7.4.4 of [3] we see that if $P$ is a Sylow $p$-subgroup of $G$ then $P=\left(P \cap G^{\prime}\right) \times(P \cap Z(N))$, where $N=N_{G}(P)$. But then if $P \cap G^{\prime} \neq 1$, we must have $P \subseteq G^{\prime}$. (Recall that $P$ is homocyclic and $G^{\prime}$, by Lemma 1(iii), must contain all the elements of order $p$. As $P \cap G^{\prime}$ is a factor of $P$, we get $P \subseteq G^{\prime}$.) Thus for each Sylow subgroup $P$, either $P \subseteq G^{\prime}$ or $P \cap G^{\prime}=1$. Hence, $G^{\prime}$ is a Hall $\pi$-subgroup for an appropriate set of primes. Note if $P \subseteq G^{\prime}$ then $P \triangleleft G$ and hence by the above $P \cap Z(G)=1$. Thus, $G^{\prime} \cap Z(G)=1$. The Schur-Zassenhaus Theorem then completes the proof.

LemMA 8. Let $G=K H$, with $K=G^{\prime}$ and $K \cap H=1$, be quasi-injective. Then, if $S$ is the Sylow p-subgroup of $K$ and $h \in H$, there is an integer $r=r(p, h)$ such that $s^{h}=s^{r}$ for all $s \in S$.

Proof. Assume that $S=\left\langle k_{1}\right\rangle \times \ldots \times\left\langle k_{n}\right\rangle$, where $\left\langle k_{i}\right\rangle$ are isomorphic cyclic subgroups. By Theorem $4,\left\langle k_{i}\right\rangle,\left\langle k_{j}\right\rangle$ and $\left\langle k_{i} k_{j}\right\rangle$ are normal subgroups of $G$ for every $i, j$. Thus there exist $r, s, t$ such that $k_{i}^{h}=k_{i}^{r}, k_{j}^{h}=k_{i}^{s}$ and $\left(k_{i} k_{j}\right)^{h}=\left(k_{i} k_{j}\right)^{t}$ with $0<r, s, t<\left|k_{i}\right|=\left|k_{j}\right|$.

But then $k_{i}^{r} k_{j}^{s}=\left(k_{i} k_{j}\right)^{h}=k_{i}^{t} k_{j}^{t}$. Hence $k_{i}^{r-t}=k_{j}^{t-s} \in\left\langle k_{i}\right\rangle \cap\left\langle k_{j}\right\rangle=1$. This implies that $r=t=s$. Thus, for every $i, k_{i}^{h}=k_{i}^{r}$. Hence the same is true for any element of $S$. (Note the result is obvious if $n=1$.)

Lemma 9. Let $G=K H$, with $K=G^{\prime}$ and $K \cap H=1$, be a quasi-injective group. If $K_{\pi}$ is the Hall $\pi$-subgroup of $K$ for some set of primes $\pi$ then $C_{H}\left(K_{n}\right)$ is a direct factor of $H$. In particular, $Z(G)=C_{H}(K)$ is a direct factor of $G$. 
Proof. It is clearly sufficient to prove that if $1 \neq x^{\mathrm{p}} \in C_{H}\left(K_{\pi}\right)$ then there is a $z \in$ $C_{\mathrm{H}}\left(K_{\pi}\right)$ such that $z^{p}=x^{p}$.

The natural map $f: K_{\pi}\left\langle x^{p}\right\rangle \rightarrow K_{\pi}$ can be extended to $F: G \rightarrow G$. Let $M=\operatorname{ker} F$; then $M \cap K_{\pi}\langle x\rangle=\left\langle x^{\text {p }}\right\rangle$ (i.e. $x \notin C\left(K_{\pi}\right)$ ) and so im $F$ contains an element $y$ of order $p$ with the same action on $K_{\pi}$ as $x$. Some conjugate $y^{k}$ of $y$ is in $H$ and still has the same action as $x$. Then $z=x\left(y^{k}\right)^{-1} \in C_{H}\left(K_{\pi}\right)$ is the required element.

The next result together with Theorem 6 and Lemmas 7, 8, 9 constitutes a characterization of the finite, quasi-injective groups.

THEOREM 10. Let $G=K H$ be such that

(i) $G^{\prime}=K$,

(ii) $K, H$ are homocyclic,

(iii) $(|K|,|H| ;=1$, $k \in K_{\text {, }}$

(iv) for each $h \in H$, if $p$ is a prime, $p|| K \mid$, there is an $r=r(p, h)$ such that $k^{h}=k^{r}$ for all

(v) $C_{H}\left(K_{\pi}\right)$ is a direct factor of $H$, where $K_{\pi}$ is a Hall $\pi$-subgroup of $K$ for some set of primes $\pi$.

Then $G$ is a quasi-injective group.

Proof. Let $L \subseteq G$ and let $f: L \rightarrow G$ be a homomorphism. We can assume, without loss of generality, that $L=(L \cap K)(L \cap H)$. Let $\pi$ be the set of primes which divide the order of $(L \cap K) /\left.\operatorname{ker} f\right|_{L \cap K}$ and choose $x$ so that $f(L \cap H) \subseteq H^{x}$.

We first note the following fact.

(I) If $h \in L \cap H$ then $f(h)=(h c)^{x}$ for some $c \in C_{H}\left(K_{\pi}\right)$.

Consider the map $f_{1}: L \rightarrow G$ defined by $f_{1}(l)=x f(l) x^{-1}$. Then $f_{1}(L \cap H) \subseteq H$ and $f_{1}$ is a homomorphism. For each $p \in \pi$, pick $k \in L \cap K$ such that $f_{1}(k)$ is a $p$-element and $f_{1}(k) \neq 1$. Then, using (iv), we have

$$
f_{1}(k)^{f_{1}(h)}=f_{1}\left(k^{h}\right)=f_{1}(k)^{h}
$$

Therefore $f_{1}(h) h^{-1}$ centralizes $f_{1}(k)$. But since $f_{1}(h) h^{-1}$ acts on $K$ by raising to powers, it must then centralize every element of order p. Hence, by Theorem 5.2.4 of [3], $f_{1}(h) h^{-1} \in C_{H}\left(K_{p}\right)$, and so $f_{1}(h) h^{-1} \in C_{H}\left(K_{\pi}\right)$, where $K_{\pi}$ is the Hall $\pi$-subgroup of $K$. Thus $f_{1}(h)=h c$, as required.

(II) We can extend $f: L \rightarrow G$ to $f_{2}: K L \rightarrow G$.

Note that $K L=K(L \cap H), f(K \cap L) \subseteq K_{\pi}$, and $\left.f\right|_{K \cap L}$ can be extended to $f_{2}: K \rightarrow K$ such that $f_{2}\left(K_{\pi}\right) \subseteq K_{\pi}$ and $f_{2}(k)=1$ if $k$ is a $\pi^{\prime}$-element. Then, if we define $f_{2}(k h)=$ $f_{2}(k) f(h)$, we can easily check, using (I), that $f_{2}$ is a homomorphism, as required.

(III) $f_{2}: K L \rightarrow G$ can be extended to $F: G \rightarrow G$.

By induction, it is sufficient to extend $f_{2}$ to $F: M \rightarrow G$, where $|M / K L|=p$. Let $h \in(M \cap H)-L$ be a $p$-element. Suppose $f_{2}\left(h^{p}\right)=\left(h^{p} c\right)^{x}$ for some $c \in C_{H}\left(K_{\pi}\right)$. The order of $c$ is less than that of $h$ and so is less than the p-part of the exponent of $H$. By (v) there is a $d \in C_{H}\left(K_{\pi}\right)$ so that $d^{p}=c$. Thus we define $F(h)=(h d)^{x}$ in order to extend $f_{2}$ to $F: M \rightarrow G$. 
The authors would like to remark that in [1] a similar characterization is given for the finite, slightly injective groups (i.e. those groups in which endomorphisms of subgroups can be extended to the whole group). It follows from this result that the class of finite quasi-injective groups is the same as the class of finite slightly injective groups.

The authors would like to express their sincere thanks to the referee for his extensive suggestions and comments. He should especially be thanked for his proof of the final result.

\section{REFERENCES}

1. D. Bertholf and G. L. Walls, Slightly injective finite groups, unpublished.

2. L. Fuchs, Infinite abelian groups I (Academic Press, 1970).

3. D. Gorenstein, Finite groups (Harper and Row, 1968).

4. B. Huppert, Endliche Gruppen I (Springer, 1967).

5. W. R. Scott, Group theory (Prentice-Hall, 1964).

Dr. Dennis Bertholf

Dr. Gary Walls

Mathematics Department

OkLahoma State University

Stillwater, OKLAHOMA 74074

SOUTHERN STATION, BOX 9265

UNIVERSITY OF SOUTHERN MisSISSIPPI

HATTIESBURG, MississiPPI 39401 